\title{
Indirect Dark Matter searches in the gamma-ray channel toward the Sun with the Fermi LAT
}

\section{F. Loparco, ${ }^{a, b, *}$ M. N. Mazziotta, ${ }^{b}$ D. Serini ${ }^{b}$ on behalf of the Fermi Large Area Telescope Collaboration}

a Dipartimento di Fisica “M. Merlin”, dell'Università e del Politecnico di Bari via Amendola 173, I-70126 Bari, Italy

${ }^{b}$ Istituto Nazionale di Fisica Nucleare, Sezione di Bari

via Orabona 4, I-70126 Bari

E-mail: francesco.loparco@ba.infn.it, marionicola.mazziotta@ba.infn.it, davide.serini@ba.infn.it

The Sun is a possible target for indirect dark matter (DM) searches, as it can gravitationally capture DM particles from the Galactic halo, which can be trapped in external orbits or sink into the solar core. We have performed a dedicated analysis of solar gamma rays collected by the Fermi Large Area Telescope (LAT) to search for possible flux excesses, which could be ascribed to DM. Gamma rays in final states of DM annihilations occurring outside the Sun can in fact reach the Earth and be detected by the LAT. Alternatively, DM particles can annihilate inside the Sun core into pairs of long-lived mediators, which are able to escape from the Sun and can decay outside the Sun, yielding gamma rays in the final state. All these processes are expected to yield an excess in the gamma-ray flux from the Sun, which appears as a specific spectral feature. Although no evidence of a DM signal has been found, we have obtained upper limits on the DM gamma-ray flux, which have been converted into constraints on the DM-nucleon scattering cross sections.

$37^{\text {th }}$ International Cosmic Ray Conference (ICRC 2021)

July 12th - 23rd, 2021

Online - Berlin, Germany

\footnotetext{
*Presenter
} 


\section{Introduction}

Although the existence of dark matter (DM) is confirmed by a variety of evidences [1], its nature is still uncertain. Weakly Interacting Massive Particles (WIMPs) are nowadays among the most popular DM candidates, since they can match nearly all DM properties inferred from experimental observations [2].

Indirect DM searches are aimed at the detection of standard model (SM) particles (gamma rays, cosmic-ray electrons and positrons, neutrinos,...) produced in the annihilations or decays of DM particles, which are gravitationally accumulated in heavy objects (targets), like galaxies or celestial bodies. A DM signature would therefore appear as an excess flux of any species of SM particles from any of these targets.

The Sun is is among the possible targets for indirect DM searches. This work will be focused on the search for possible gamma-ray signals originated from DM annihilations in the solar environment, using the data collected by the Fermi Large Area Telescope (LAT) [3]. We will search for particular spectral features, related to the specific processes described in sec. 2. The analysis procedure will be illustrated in sec. 3 and the results will be discussed in sec. 4 .

\section{Gamma rays from dark matter annihilations in the Sun}

DM particles from the galactic halo can be gravitationally trapped by the Sun through scatterings with the nuclei in the solar environment.

DM particles $\chi$ can be captured by the Sun in external orbits, thus forming a halo around the Sun, and then annihilate outside the Sun into pairs of SM particles, including gamma rays, that can reach the Earth and be detected. In case of DM particles annihilating directly into gamma-ray pairs $(\chi \chi \rightarrow \gamma \gamma)$, the energy spectrum of gamma rays would exhibit a line-like shape at an energy corresponding to the mass of the DM particle $m_{\chi}$.

Alternatively, DM particles can lose their energy through subsequent scatterings with the solar nuclei, until they reach the Sun core, where they can annihilate into pairs of SM particles. However, almost all SM particles produced in these processes, with the exception of low-energy neutrinos, are not able to escape from the Sun. However, a detectable signal at Earth would be possible if pairs of DM particles in the solar core could annihilate into pairs of neutral long-lived mediators $\phi$, which are able to escape from the Sun and then decay into pairs of SM particles [4-10]. In this case, gamma rays would be produced at the end of the reaction chain $\chi \chi \rightarrow \phi \phi, \phi \rightarrow \gamma \gamma$. If DM particles annihilate at rest and if the mediators are light $\left(m_{\phi} \ll m_{\chi}\right)$, the gamma-ray spectrum is expected to exhibit a box-like shape, with an upper edge at an energy corresponding to the mass of the DM particle $m_{\chi}$.

In both scenarios, the number of DM particles in the Sun at a given time $N_{\chi}(t)$ can be evaluated by solving by the following balance equation:

$$
\frac{d N_{\chi}}{d t}=\Gamma_{\text {cap }}-C_{\text {ann }} N_{\chi}^{2}
$$

where $\Gamma_{\text {cap }}$ is the DM capture rate and $C_{\text {ann }}$ is the DM annihilation factor, which depends on the annihilation cross section. In eq. 1 we have not included the evaporation mechanism, which is negligible for DM masses above a few $\mathrm{GeV}[11,12]$. 
At equilibrium, i.e. when $d N_{\chi} / d t=0$, the annihilation rate is independent of the annihilation cross section and is set by the capture rate $\Gamma_{\text {cap }}$ :

$$
\Gamma_{\mathrm{ann}}=\frac{1}{2} C_{\mathrm{ann}} N_{\chi}^{2}=\frac{1}{2} \Gamma_{\text {cap }}
$$

The factor $1 / 2$ in the previous equation accounts for the two DM particles involved in each annihilation event. The capture rate, which depends on the scattering cross section (either spin-dependent or spin independent), on the local halo DM number density $\rho_{\odot}$, on the DM mass $m_{\chi}$, on the DM velocity distribution and on its dispersion. In this work we have evaluated the capture rate using the DARKSUSY code version 6.1.0 [13-15] with its default settings, i.e. a local DM density $\rho=0.3 \mathrm{GeV} / \mathrm{cm}^{3}$, a DM-nucleon scattering cross section $\sigma=10^{-40} \mathrm{~cm}^{2}$ (in both the spindependent and spin-independent cases) and a Maxwellian velocity distribution for DM particles with average velocity of $220 \mathrm{~km} / \mathrm{s}$ and dispersion of $270 \mathrm{~km} / \mathrm{s}$. In particular, the dependence of the capture rate on the DM-nucleon cross section is linear.

If equilibrium between capture and annihilation is not reached, the general solution of eq. 1 is given by:

$$
N_{\chi}(t)=\sqrt{\frac{\Gamma_{\text {cap }}}{C_{\text {ann }}}} \tanh \left(\frac{t}{\tau}\right)
$$

where $\tau=\left(\Gamma_{\text {cap }} C_{\text {ann }}\right)^{-1 / 2}$ [16] is the equilibrium time scale of the process. In this case, the annihilation rate in eq. 2 should be multiplied by the factor $\tanh ^{2}(t / \tau)$.

In the mediator scenario, the DM gamma-ray flux at Earth is given by [17]:

$$
\Phi_{\mathrm{DM}}(E)=N_{\gamma}(E) \frac{\Gamma_{\text {cap }}}{4 \pi D^{2}}\left(e^{-R_{\odot} / L}-e^{-D / L}\right)
$$

where $R_{\odot}$ is the solar radius, $D$ is the Sun-Earth distance, $L$ is the $\phi$ decay length and $N_{\gamma}(E)$ is the spectrum of photons produced by the mediator decays. Since the process $\phi \rightarrow \gamma \gamma$ is a two-body decay, $N_{\gamma}(E)$ will have a box-like shape and, if $m_{\phi} \ll m_{\chi}$, the box will extend from $E=0$ to $E=m_{\chi}$.

\section{Analysis of the Fermi LAT data}

The analysis presented in this work has been performed using a set of P8R3_CLEAN photon events [18] collected by the Fermi LAT during its first 10 years of operation. Full details on the analysis procedure are given in ref. [19].

The analysis is performed using an on/off technique, in which the data from a Region of Interest (RoI) centered on the Sun ("on region") are combined with those from a RoI centered on the anti-Sun ("off region"), whose position at any time is defined as the position that the Sun will take 6 months later. The "on" and the "off" regions are defined as disks of $2^{\circ}$ angular radius, centered on the Sun and on the anti-Sun respectively. We selected photons with energies above $100 \mathrm{MeV}$ collected in the time intervals when the LAT was operating in its standard science operation configuration and was outside the South Atlantic Anomaly (SAA). We excluded from the analysis the time intervals in which solar flares were registered. To avoid contamination from the Earth limb we also discarded data taken during the times in which the separation between the center of the "on" ("off") RoI 
direction and the zenith exceed $78^{\circ}$. We also selected time intervals when the RoIs were observed with off-axis angles in the instrument frame smaller than $64.5^{\circ}$. Finally, we required that the Sun (anti-Sun) should be at galactic latitude $|b|>5^{\circ}$ and at a minimum angular separation of $4^{\circ}$ from the Moon and from any bright source in the 3FGL catalog [20].

We have implemented a Poisson maximum likelihood fitting procedure to search for possible local excesses in the count spectra of photons from the "on" region, with a line-like or box-like shape. When folded with the instrument energy response function, these features should appear as a broad peak or a smooth edge respectively, with a width determined by the energy resolution of the LAT. The fits are performed in sliding energy windows from $100 \mathrm{MeV}$ to $150 \mathrm{GeV}$. Each energy window is defined as the interval $\left[(1-w) E_{\mathrm{w}},(1+w) E_{\mathrm{w}}\right]$, where $E_{\mathrm{w}}$ is the energy corresponding to the center of the window and the parameter $w$, which define the width of the windows, has been set to 0.6 to account for the LAT energy resolution.

The gamma-ray fluxes in the "on" and the "off" regions are modeled as follows:

$$
\begin{gathered}
\Phi^{O N}(E)=\Phi_{\text {smooth }, b k g}(E)+\Phi_{\text {smooth }, \text { sig }(E)}+\Phi_{\text {feat }}^{\text {ON }}(E)+\Phi_{\text {feat }}^{\text {extra }}(E) \\
\Phi^{\text {OFF }}(E)=\Phi_{\text {smooth }, b k g}(E)+\Phi_{\text {feat }}^{\text {extra }}(E) .
\end{gathered}
$$

The term $\Phi_{\text {smooth,bkg }}(E)$ in the previous equations represents the background flux (diffuse emission, unresolved point sources, cosmic-ray events misclassified as photons, etc.), which appears in both the "on" and the "off" regions and is described by a simple power law; the term $\Phi_{\text {smooth,sig }}(E)$ represents the gamma-ray flux from the steady solar emission, which appears only in the "on" region and is also described by a simple power law; the term $\Phi_{f \text { eat }}^{O N}(E)$ represents the possible spectral feature originated from solar DM, which apperars only in the "on" region; finally the term $\Phi_{\text {feat }}^{\text {extra }}(E)$ represents a possible feature originated from instrumental systematic effects, which should appear in both the "on" and the "off" regions, with the same shape as the DM-induced feature. In the case of the search for line-like features, the features are described by a Dirac-delta function $\delta\left(E-E_{w}\right)$, while in the case of box-like features they are described by a Heaviside step function $\theta\left(E_{w}-E\right)$.

The expected photon counts in each energy bin of the "on" and "off" regions can be evaluated by folding the flux models with the instrument response functions and taking exposures into account. We assume that the observed counts in each bin are independent and Poisson distributed. We can therefore define the likelihood function:

$$
\mathcal{L}=\mathcal{L}_{o n} \times \mathcal{L}_{\text {off }}=\prod_{j=1}^{N} \mathrm{e}^{-\mu_{\mathrm{j}}^{\text {on }}} \frac{\left(\mu_{\mathrm{j}}^{\text {on }}\right)^{\text {no }}}{\mathrm{n}_{\mathrm{j}}^{\text {on }} !} \times \prod_{\mathrm{j}=1}^{\mathrm{N}} \mathrm{e}^{-\mu_{\mathrm{j}}^{\text {off }}} \frac{\left(\mu_{\mathrm{j}}^{\text {off }}\right)^{\mathrm{n}_{\mathrm{j}}^{\text {off }}}}{\mathrm{n}_{\mathrm{j}}^{\text {off }} !} .
$$

For both line-like and box-like features we have tested the hypothesis of a signal (i.e. $\Phi_{\text {f eat }}^{O N}>0$ ) against the null hypothesis (i.e. $\Phi_{f e a t}^{O N}=0$ ). The parameters of each fit are evaluated using the MINUIT code within the ROOT toolkit [21, 22]. The local test statistic is $T S=-2\left(\log \mathcal{L}_{0, \text { max }}-\right.$ $\log \mathcal{L}_{1, \text { max }}$ ), where $\mathcal{L}_{0, \text { max }}$ and $\mathcal{L}_{1, \text { max }}$ are the values of the likelihood functions obtained when fitting the data with the models corresponding to the null hypothesis and the signal hypothesis, respectively. Since the two models are nested and differ by one parameter (the intensity of the feature in the "on" region), the TS obeys a $\chi^{2}$ distribution with one degree of freedom. We have therefore evaluated the $95 \%$ confidence limits (CL) on the intensity of the feature by setting $\Delta \log \mathcal{L}=\log \mathcal{L}_{\text {max }}-2.71 / 2$. 

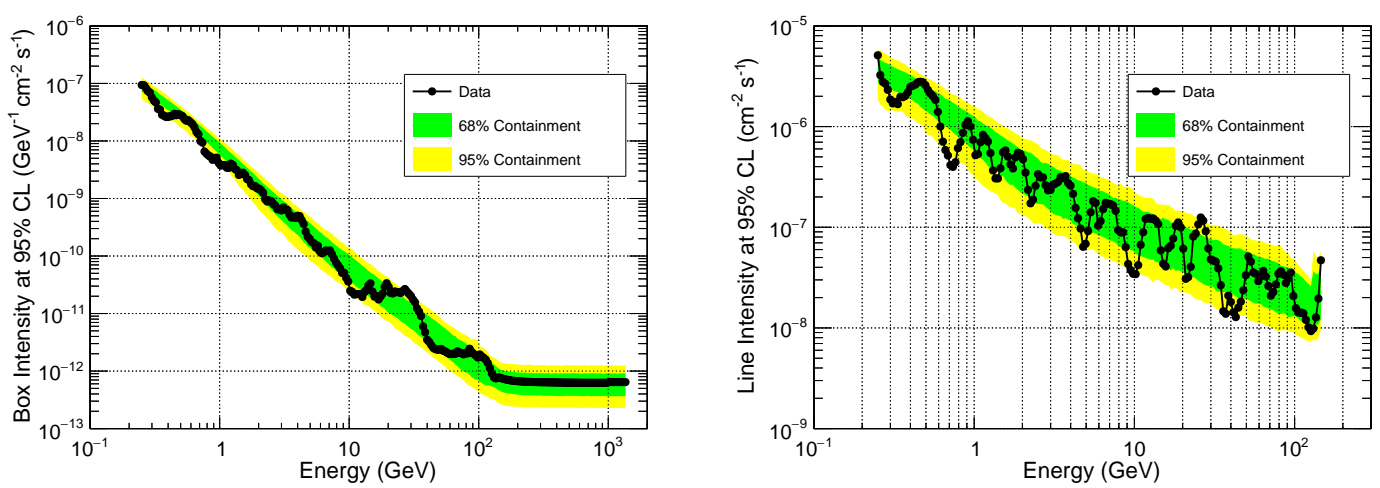

Figure 1: Upper limit at 95\% confidence level on the intensity of the feature in the solar spectrum. Left panel: box model; Right panel: line model. The green and yellow regions indicate the central $68 \%$ and $95 \%$ expectation bands for the $95 \%$ CL limits evaluated from the pseudo-experiments.

To evaluate the expectation bands for our results, i.e. the sensitivity to the null hypothesis, we have implemented a set of pseudo-experiment in which the gamma-ray fluxes in the "on" and "off" regions are modeled with a smooth power law template. The counts in each energy bin are extracted from Poisson distributions with mean values obtained from the template models. The same fit procedure as for real data is applied to each pseudo-experiment, and the distributions of the fitted parameters and of the $T S$ are built. The containment bands for the parameters and for the $T S$ are then evaluated as the quantiles of these distributions.

All possible features are found to be insignificant. Fig. 1 shows the upper limits (ULs) at $95 \%$ confidence level on the intensity of the box-like and of the line-like features respectively. The plots central $68 \%$ and $95 \%$ containment bands for the ULs, evaluated from the pseudo-experiments are also shown. We see that in both cases the measured upper limits lie within the central $95 \%$ expectation band.

\section{Limits on the dark matter scattering cross section}

In the case of the mediator scenario, the limits on the intensity of the features can be converted into limits on the flux at Earth $\Phi_{\mathrm{DM}}(E)$ of gamma rays from DM, which in turn can be converted into limits on the DM-nucleon cross section. As discussed in Sec.2, we have calculated the capture rate with the DARKSUSY code with a reference cross section of $10^{-40} \mathrm{~cm}^{2}$. The upper limit on the cross section is therefore given by:

$$
\sigma_{\mathrm{UL}}\left(m_{\chi}\right)=\frac{\Phi_{\mathrm{UL}}\left(E=m_{\chi}\right)}{\Phi_{\mathrm{DM}}\left(E=m_{\chi}\right)} \times 10^{-40} \mathrm{~cm}^{2} .
$$

On the other hand, for the inelastic scattering scenario, we are not able to perform the calculation of the capture rate, since at present all available models in the literature start from energies of about $100 \mathrm{GeV}$, and are therefore valid above the energy range explored in our analysis [25].

Figure 2 shows the ULs at 95\% CL on the DM-nucleon scattering cross sections (spindependent and spin-independent) in both the elastic and inelastic case. These limits have been 


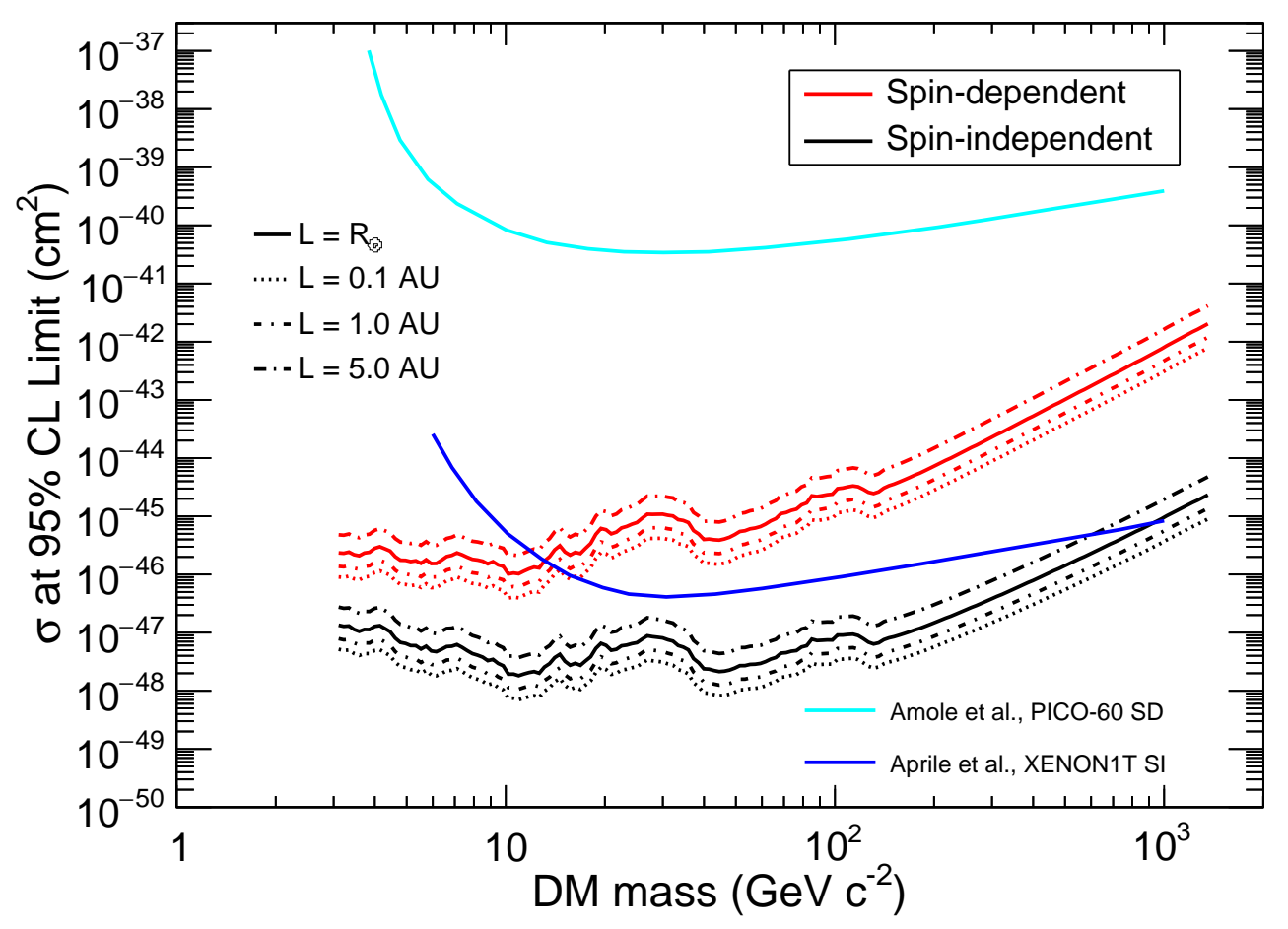

Figure 2: Limits at $95 \% \mathrm{CL}$ on the DM-nucleon cross section via long-lived mediator for four different decay lengths $L=R_{\odot}, 0.1,1$ and 5 AU. The limits at 90\% CL from the PICO-60 experiment [23] in the case of spin-dependent (SD) scattering (cyan line) and from the XENON1T experiment [24] in the case of spin-independent (SI) scattering (blue line) are also shown.

calculated for four values of the decay length of the intermediate state, between 0.1 and 5 AU. Our limits are consistent with those obtained from direct measurements of the spin-independent and of the spin-dependent DM-nucleon cross sections performed by the XENON1T [24] and PICO-60 [23] experiments respectively.

The results in Fig.2 have been obtained assuming equilibrium between DM capture and annihilation in the Sun. This condition is met only if the equilibrium time scale $\tau$ is less than the age of the Sun. However, if equilibrium is not reached, the annihilation rate is reduced of a factor $\tanh ^{2}(t / \tau)$, and consequently the limits on the DM-nucleon cross sections must be properly rescaled. In Fig. 3 the limits on the spin-dependent cross section in the equilibrium setup are shown together with those in a non-equilibrium setup, in which we have assumed a velocity-averaged cross section at the level of the thermal relic value $\left\langle\sigma_{a n n} v\right\rangle=3 \times 10^{-26} \mathrm{~cm}^{3} / \mathrm{s}$ [26]. We see that the limits evaluated with the non-equilibrium assumption are weaker than those evaluated assuming equilibrium, but the differences are negligible for DM masses in the $\mathrm{TeV}$ region.

\section{Conclusions}

We have analyzed a 10-years dataset collected by the Fermi LAT to search for possible linelike or box-like features in the energy spectrum of gamma rays from the Sun, which could be originated from the annihilations of DM particles in the solar environment. Although our search 




Figure 3: Upper limits at 95\%CL on the spin-dependent DM-nucleon scattering cross section for the longlived mediator with decay length $L=R_{\odot}$. We also show the limits from the HAWC and Fermi [12] with gamma rays from DM and the Fermi-LAT results obtained with cosmic-ray electrons [17]. The correction for non-equilibrium with $\left\langle\sigma_{a n n} v\right\rangle=3 \times 10^{-26} \mathrm{~cm}^{3} / \mathrm{s}$ is also shown.

yielded negative results, we were able to set limits on the intensities of possible feature, which were converted into limits on the DM-nucleon scattering cross sections. For DM masses up to about $150 \mathrm{GeV} / \mathrm{c}^{2}$, the limits are in the range $10^{-46}-10^{-45} \mathrm{~cm}^{2}$ for the spin-dependent scattering and in the range $10^{-48}-10^{-47} \mathrm{~cm}^{2}$ for the spin-independent case, and are consistent with constraints from other experiments.

\section{Acknowledgements}

The Fermi-LAT Collaboration acknowledges support for LAT development, operation and data analysis from NASA and DOE (United States), CEA/Irfu and IN2P3/CNRS (France), ASI and INFN (Italy), MEXT, KEK, and JAXA (Japan), and the K.A. Wallenberg Foundation, the Swedish Research Council and the National Space Board (Sweden). Science analysis support in the operations phase from INAF (Italy) and CNES (France) is also gratefully acknowledged. This work performed in part under DOE Contract DE-AC02-76SF00515.

\section{References}

[1] P. A. Zyla et al. [Particle Data Group], Prog. Theor. Exp. Phys. 2020, 083 C01 (2020)

[2] L. Roszkowski et al., Rep. Prog. Phys. 81 (2019) 066201 
[3] W. B. Atwood et al., Astrophys. J. 697 (2009), 1071-1102

[4] M. Pospelov et al., Phys. Lett. B662 (2008), 53-61

[5] N. Arkani-Hamed et al., Phys. Rev. D79 (2009), 015014

[6] P. Schuster et al. Phys. Rev. D81 (2010), 016002

[7] P. Schuster et al. Phys. Rev. D82 (2010), 115012

[8] R. K. Leane et al., Phys.Rev. D95 (2017), 123016

[9] N. F. Bell and K. Petraki, JCAP 1104 (2011), 003

[10] C. Arina et al., Phys. Rev. D96 (2017), 063010

[11] K. Griest and D. Seckel, Nucl. Phys B283 (1987), 681-705

[12] A. Albert et al., Phys. Rev. D98 (2018), 123012

[13] P. Gondolo et al., JCAP 0407 (2004), 008

[14] T. Bringmann et al., JCAP 1807 (2018), 033

[15] http://www . darksusy . org/

[16] G. Jungman et al., Phys. Rep. 267 (1996), 195-373

[17] A. Cuoco et al., Phys. Rev. D101 (2020), 022002

[18] W. Atwood et al., arXiv 13013.3514 (2013)

[19] M. N. Mazziotta et al., Phys. Rev. D102 (2020), 022003

[20] F. Acero et al., Astrophys. J. Suppl. 218 (2015), 23

[21] R. Brun and F. Rademakers Nucl. Instrum. Meth. A389 (1997), 81-86

[22] https://root.cern.ch/

[23] C. Amole et al., Phys. Rev. D100 (2019), 022001

[24] E. Aprile et al., Phys. Rev. Lett. 121 (2018), 111302

[25] A. Menon et al., Phys. Rev. D82 (2010), 015011

[26] G. Steigman et al. Phys. Rev. D86 (2012), 023506 